\title{
Practical approximations for multivariate characteristics of risk processes
}

\author{
M.A. Usábel ${ }^{\mathrm{a}, \mathrm{b}, *}$ \\ ${ }^{a}$ Dept. of Actuarial Mathematics and Statistics, Heriot-Watt University, Riccarton, Edinburgh EH14 4AS, UK \\ b Dep. Economía de la Impresa, Universidad Carlos III. Campus de Colmenarejo, Madrid, Spain
}

\begin{abstract}
The applicability aspects of power series expansions with respect to the arrival intensity, based on recursive algorithms, when approximating multivariate finit time ruin probabilities will be substantially enhanced using double Laplace transforms.

We will also prove that power series methodology may be considered as an outstanding complement to diffusion approximations when the time horizon considered is not large.
\end{abstract}

Keywords: Recursive procedures; Double Laplace transforms; Finite time multivariate ruin probability; Multiple integrals; Power series expansions

\section{Introduction}

Definin a classical risk process in continuous time $\left\{Z_{t}\right\}_{t \geq 0}$ with $U_{k}$ claim sizes and premium $c$ per time unit,

$$
Z_{t}=u+c t-\sum_{k=1}^{N_{t}} U_{k},
$$

where $u$ are the initial reserves and $N_{t}$ the total number of claims up to time $t$ (with $\mathrm{d}$ f. of the waiting times between claims $w(t)$ ), where $\lambda$ is the average number of claims in one year. Let $B$ denote the distribution function of claim sizes $U_{k}$ with mean $\mu^{-1}$ and $c=\lambda \mu^{-1}(1+\theta)$, where $\theta$ is the premium loading factor.

Let us now defin $\tau=\inf \left\{w>0: Z_{w}<0\right\}$ as the ruin time and $Y=-Z_{\tau}$ as the defici at ruin time or severity of ruin and $X=Z_{\tau^{-}}$as the surplus just before ruin.

If we consider a time horizon of $t$ years (or time units), the finit time multivariate probability of ruin with initial reserves $u$, arrival intensity $\lambda$ and severity of ruin less than $y$ and surplus less than $x$ is define as

$$
P\{\tau<t, X \leq x, Y \leq y\}=\Psi_{t, u, x, y}(\lambda) .
$$

\footnotetext{
in Presented at the 2nd IME Conference, Lausanne, 1998. The author gratefully acknowledges the financia support from: Bacas de Perfeccionamiento de Doctores en el Extranjero, 1999, MEC, Spain.

* Tel.: +34-91-619-8917; fax: +34-91-571-0114

E-mail address: ausabel@correo.cop.es (M.A. Usábel)
} 
When the time horizon is infinite the multivariate ultimate ruin probability can be expressed as

$$
P\{\tau<\infty, X \leq x, Y \leq y\}=\Psi_{u, x, y}(\lambda) .
$$

Frey and Schmidt (1996) used the following Taylor-series expansion (1.2) for the probability of ruin with time span $t, \Psi_{t, u, x, y}$ when the classical case of risk theory is considered, $w(t)=\lambda \mathrm{e}^{-\lambda t}$, restricted to the case when the premium loading factor was defined

$$
\begin{aligned}
& \theta=\frac{1-\lambda \mu^{-1}}{\lambda \mu^{-1}}, \quad \theta>0, \\
& \Psi_{t, u, x, y}(\lambda)=\sum_{k=1}^{\infty} \frac{\Psi_{t, u, x, y}^{\prime(k)}(0)}{k !} \lambda^{k}, \quad \lambda \geq 0,
\end{aligned}
$$

they proved that the function is analytic at $\lambda=0$ and the Taylor-series expansion (1.2) has an infinit radius of convergence. They also specifie a recursive formula to obtain the nth derivative at $\lambda=0$ (Theorem 2 of the original paper by Frey and Schmidt, 1996).

Theorem 1 (Frey and Schmidt). For each $k \geq 1,0 \leq u, t<\infty, 0<x, y \leq \infty$, it holds that

$$
\frac{\Psi_{t, u, x, y}^{\prime(k)}(0)}{k !}=\frac{\Psi_{u, x, y}^{\prime(k)}(0)}{k !}-\sum_{l=1}^{k} q_{t, u, x, y}^{(k-l, l)},
$$

where the quantities $q_{t, u, x, y}^{(n, l)}$ are given recursively by

$$
q_{t, u, x, y}^{(n, l)}=\int_{0}^{t}\left(\int_{0}^{u+s} q_{t-s, u+s-z, x, y}^{(n-1, l)} \mathrm{d} B(z)-q_{t-s, u+s, x, y}^{(n-1, l)}\right) \mathrm{d} s
$$

and

$$
q_{t, u, x, y}^{(0, l)}=\frac{\Psi_{u+t, x, y}^{\prime(l)}(0)}{l !}=F_{x, y} * G^{*(l-1)}(u+t) .
$$

They also proved (in Theorem 1 of the original paper) that

$$
\frac{\Psi_{z, x, y}^{\prime(l)}(0)}{l !}=F_{x, y} * G^{*(l-1)}(z),
$$

where $G(w)=\int_{0}^{w}(1-B(z)) \mathrm{d} z$ and introduce the functions

$$
F_{x, y}^{1}(w)=\int_{w}^{\max \{x, w\}}(B(z+y)-B(z)) \mathrm{d} z=\int_{w}^{\max \{x, w\}}(g(z)-g(z+y)) \mathrm{d} z, \quad x>0,
$$

or when $X=Z_{\tau^{-}}$, the surplus just before the ruin is define as $u+x(x>0)$,

$$
F_{x, y}^{2}(w)=\int_{w}^{w+x}(B(z+y)-B(z)) \mathrm{d} z=\int_{w}^{w+x}(g(z)-g(z+y)) \mathrm{d} z,
$$

in this last case the above mentioned surplus should be always greater than the initial reserves $u$.

Using the results by Frey and Schmidt (1996), it can be proved, Usabel (1999), that 
Theorem 2 (Usábel). In the conditions stated for Theorem 1,

$$
\frac{\Psi_{t, u, x, y}^{\prime(k)}(0)}{k !}=\frac{\Psi_{u, x, y}^{\prime(k)}(0)}{k !}-\left(\sum_{l=0}^{k-1}(-1)^{l} \frac{t^{l}}{l !} \mathcal{F}_{t, u, x, y}^{k-l}\right),
$$

where

$$
\begin{aligned}
\mathcal{F}_{t, u, x, y}^{1} & =F_{x, y}(u+t), \quad \mathcal{F}_{t, u, x, y}^{k}=\int_{0}^{t} \int_{0}^{u+s} \mathcal{F}_{t-s, u+s-z, x, y}^{k-1} b(z) \mathrm{d} z \mathrm{~d} s+F_{x, y} * G^{*(k-1)}(u+t), \\
k & =2,3, \ldots
\end{aligned}
$$

Other papers where power-series expansions with respect to $\lambda$, arrival intensity, have been used in approximating probability characteristics of stochastic models driven by a Poisson process are Hooghiemstra et al. (1988), Rieman and Simon (1988), Blanc (1991), Simon (1993) and Kroese and Schmidt (1995).

Maclaurin's polynomial expansion (1.2) constitutes an original and alternative approach in approximating multivariate characteristics of Classical Ruin processes other than inversion of Laplace transforms for particular claim size distributions - Gerber et al. (1987) and Dufresne and Gerber (1988) (a), (b) — or discretization of the claim size and time — Dickson (1989), Dickson and Waters (1992), Dickson (1993) — applying the so-called Panjer's recursive algorithm (Panjer, 1981).

Nevertheless, the practical application of the polynomial expansion approach, implemented using formulas (1.3) or (1.8), is quite limited due to the complexity involved in approximating the recursive double integrals stated in formulas (1.4) and (1.9) respectively.

In the present work, we will first in Section 2, introduce an interesting result regarding the double Laplace transform of certain families of functions generated by recursive double integrals: Theorem 3 . The proof is presented in Appendix A.

In Section 3, we will see that the double Laplace transform of the recursive functions, see Theorem 1 or 2, involved in the calculation of the coefficient of the Taylor expansion of the multivariate finit time ruin probability function can be obtained easily using the results from Theorem 3 .

The practical algorithm to implement this methodology is studied in Section 4. Questions regarding the computational efficien y of this approach are answered in Section 4. Section 5 contains a numerical illustration considering Pareto claim sizes and concluding comments are introduced in Section 6.

We also have to mention that the problem of ruin in the collective risk theory has been extensively treated in actuarial literature using integral transforms. Since the paper by Sparre Andersen in 1955 many authors developed approximations for the ruin probability using Laplace-Stieltjes transforms. Cramer (1955) used the Wiener-Hopf for the classical case and Thorin $(1970,1971,1973,1977)$ introduced the generalization when epochs of claims form a renewal process. Thorin and Wikstad (1973), Wikstad $(1971,1977)$ used the Piessens (1969) inversion method of the Laplace transforms and Bohman $(1971,1974,1975)$ focussed on inversions of Fourier transforms and Seal $(1971,1974)$ dealt with both Laplace and Fourier numerical inversions. Seal (1977) obtained an interesting result for the classical case and exponential claim size distribution using the Bromwich-Mellin inversion formula for Laplace transforms. Nevertheless, the cited actuarial literature did not contemplate the multivariate case and just focussed on the simpler and particular case when $X=\infty$ and $Y=\infty$.

\section{Laplace transforms}

Let us defin the so-called double Laplace transform operator of a general function $H_{t, u, x, y}^{k}$,

$$
L\left(H_{t, u, x, y}^{k} ;(m, n)\right)=H_{m, n, x, y}^{k * *}=\int_{t=0}^{\infty} \int_{u=0}^{\infty} H_{t, u, x, y}^{k} \mathrm{e}^{-m t-n u} \mathrm{~d} u \mathrm{~d} t
$$


and the simple Laplace transform

$$
L\left(D_{x, y}(u) ; s\right)=D_{x, y}^{*}(s)=\int_{u=0}^{\infty} D_{x, y}(u) \mathrm{e}^{-s u} \mathrm{~d} u .
$$

Subsequently, the inverse Laplace transform operators are presented as

$$
\operatorname{Invd}\left\{L\left(H_{t, u, x, y}^{k} ;(m, n)\right) ;(t, u)\right\}=H_{t, u, x, y}^{k}, \quad \operatorname{Inv}\left\{L\left(D_{x, y}(u) ; s\right) ; u\right\}=D_{x, y}(u) .
$$

Remark 1. Notice that using the notation

$$
H_{m, u, x, y}^{k *}=\int_{t=0}^{\infty} H_{t, u, x, y}^{k} \mathrm{e}^{-m t} \mathrm{~d} t
$$

we get

$$
\left.H_{m, u, x, y}^{k *}=\operatorname{Inv}\left\{H_{m, n, x, y}^{k * *} ; u\right) ; u\right\}
$$

and

$$
H_{t, u, x, y}^{k}=\operatorname{Inv}\left\{H_{m, u, x, y}^{k *} ; t\right\} .
$$

Let us now introduce the following theorem regarding the double Laplace transform of recursive functions. The proof is presented in Appendix A.

Theorem 3. Let us consider a certain family of functions, $D_{x, y}^{k}(z)(k=1,2, \ldots)$ and $b(z)$, with infinitel differentiable Laplace transforms.

If a set of functions $H_{t, u, x, y}^{k}(k=1,2, \ldots)$ can be obtained using the former family and the following recursive expression

$$
\begin{aligned}
H_{t, u, x, y}^{k+1}= & \int_{s=0}^{t} \int_{z=0}^{u+s} H_{t-s, u+s-z, x, y}^{k} b(z) \mathrm{d} z \mathrm{~d} s-\int_{s=0}^{t} H_{t-s, u+s, x, y}^{k} \mathrm{~d} s+D_{x, y}^{k+1}(u+t)=\mathcal{I}_{1}\left(H_{t, u, x, y}^{k}\right) \\
& -\mathcal{I}_{2}\left(H_{t, u, x, y}^{k}\right)+D_{x, y}^{k+1}(u+t)
\end{aligned}
$$

with initial condition

$$
H_{t, u, x, y}^{1}=D_{x, y}^{1}(u+t),
$$

then the double Laplace transform of this set of functions can be expressed also recursively, for $n \neq m$,

$$
H_{m, n, x, y}^{(k+1) * *}=\frac{H_{m, n, x, y}^{k * *}\left(b^{*}(n)-1\right)-H_{m, m, x, y}^{k * *}\left(b^{*}(m)-1\right)}{m-n}+\frac{D_{x, y}^{(k+1) *}(n)-D_{x, y}^{(k+1) *}(m)}{m-n}
$$

with initial condition

$$
H_{m, n, x, y}^{1 * *}=\frac{\left(D_{x, y}^{1 *}(n)-D_{x, y}^{1 *}(m)\right)}{m-n}
$$

and for $n=m$,

$$
H_{m, m, x, y}^{k * *}=\frac{Z_{k}^{k}(m)}{(-1)^{k} k !},
$$


where also recursively

$$
\begin{aligned}
Z_{l}^{j}(m) & =\sum_{i=l-1}^{j}\left(\begin{array}{l}
j \\
i
\end{array}\right) Z_{l-1}^{i}(m)\left(b^{*}(m)-1\right)^{\prime(j-i)}+\left(\frac{(-1)^{l-1} j !}{(j-(l-1)) !}\right)\left(D_{x, y}^{l *}(m)\right)^{\prime(j-(l-1))}, \\
l & =1,2,3, \ldots, \quad j=l, l+1, \ldots,
\end{aligned}
$$

and

$$
Z_{1}^{j}(m)=\left(D_{x, y}^{1 *}(m)\right)^{\prime(j)}, \quad j=1,2, \ldots
$$

\section{Maclaurin's expansion of the multivariate ruin probability}

The Maclaurin's expansion with respect to the arrival intensity for the finit time multivariate ruin probability (1.2) can be obtained using either Theorem 1 or 2 .

Regarding Theorem 1, however, a most straightforward formula can be obtained introducing the function

$$
\Xi_{t, u, x, y}^{k}=\sum_{l=1}^{k} q_{t, u, x, y}^{(k-l, l)}
$$

then it is not hard to prove from (1.3),(1.4) and (1.5) that

$$
\begin{aligned}
\Xi_{t, u, x, y}^{1}= & q_{t, u, x, y}^{(0,1)}=F_{x, y}(u+t), \quad \Xi_{t, u, x, y}^{k}=\sum_{l=1}^{k} q_{t, u, x, y}^{(k-l, l)}=\mathcal{I}_{1}\left(\sum_{l=1}^{k-1} q_{t, u, x, y}^{((k-1)-l+1, l)}\right) \\
& -\mathcal{I}_{2}\left(\sum_{l=1}^{k-1} q_{t, u, x, y}^{((k-1)-l+1, l)}\right)+q_{t, u, x, y}^{(0, k)}=\mathcal{I}_{1}\left(\Xi_{t, u, x, y}^{k-1}\right)-\mathcal{I}_{2}\left(\Xi_{t, u, x, y}^{k-1}\right)+F_{x, y} * G^{*(k-1)}(u+t), \\
k= & 2,3, \ldots
\end{aligned}
$$

Using Theorem 2 instead the recursive formulas unfold easier (see (1.9))

$$
\mathcal{F}_{t, u, x, y}^{1}=F_{x, y}(u+t), \mathcal{F}_{t, u, x, y}^{k}=\mathcal{I}_{1}\left(\mathcal{F}_{t, u, x, y}^{k-1}\right)+F_{x, y} * G^{*(k-1)}(u+t), \quad k=2,3, \ldots
$$

The $k$ th derivative at zero of the finit time multivariate ruin probability function with respect to the arrival intensity $\lambda$ is either

$$
\frac{\Psi_{t, u, x, y}^{\prime(k)}(0)}{k !}=F_{x, y} * G^{*(k-1)}(u)-\Xi_{t, u, x, y}^{k}=F_{x, y} * G^{*(k-1)}(u)-\sum_{l=0}^{k-1}(-1)^{l} \frac{t^{l}}{l !} \mathcal{F}_{t, u, x, y}^{k-l}, \quad k=1,2, \ldots
$$

Theorem 3 can then be applied to the recursive expressions (3.1) and (1.9), and obtain also recursively the double Laplace transforms

$$
\left\{\Xi_{t, u, x, y}^{k * *}\right\}, \quad\left\{\mathcal{F}_{t, u, x, y}^{k * *}\right\}, \quad k=1,2,3, \ldots,
$$

where in both cases

$$
D^{k}(z)=F_{x, y} * G^{*(k-1)}(z), \quad D^{k *}(z)=F_{x, y}^{*}(s)\left(g^{*}(s)\right)^{k-1}, \quad k=1,2,3, \ldots
$$

are known functions presented in Section 1 along with $b(z)$, density function of the claim sizes considered. 
Remark 2. Notice that, as long as the recursive formula for the family of functions $\mathcal{F}_{t, u, x, y}^{k}$ is simpler, the subsequent recursive expressions involved in the calculation of $\mathcal{F}_{t, u, x, y}^{k * *}$, using Theorem 3 , are always performed considering $b^{*}(m)$ instead of $\left(b^{*}(m)-1\right)$. This can be easily noticed looking at the proof of Lemma 2 in Appendix A.

Finally, the finit time multivariate ruin probability can be expanded as a Maclaurin's series using inverse Laplace transforms

$$
\begin{aligned}
& \Psi_{t, u, x, y}(\lambda)=\sum_{k=1}^{\infty}\left(\operatorname{Inv}\left\{F_{x, y}^{*}(s)\left(g^{*}(s)\right)^{k-1} ; u\right\}-\operatorname{Invd}\left\{\Xi_{m, n, x, y}^{k * *} ;(t, u)\right\}\right) \lambda^{k}, \\
& \Psi_{t, u, x, y}(\lambda)=\sum_{k=1}^{\infty} \operatorname{Inv}\left\{F_{x, y}^{*}(s)\left(g^{*}(s)\right)^{k-1} ; u\right\} \lambda^{k}-\sum_{k=1}^{\infty}\left(\sum_{l=0}^{k-1}(-1)^{l} \frac{t^{l}}{l !} \operatorname{Invd}\left\{\mathcal{F}_{m, n, x, y}^{(k-l) * *} ;(t, u)\right\}\right) \lambda^{k} .
\end{aligned}
$$

\section{Practical algorithm}

It is clear that the main concern is to approximate the coefficient of the Taylor expansion

$$
\Psi_{t, u, x, y}(\lambda) \simeq \Psi_{t, u, x, y}^{L}(\lambda)=\sum_{k=1}^{L} \frac{\Psi_{t, u, x, y}^{\prime(k)}(0)}{k !} \lambda^{k}
$$

for a truncated series of order $L$. It was proved in Theorems 1 and 2 that the derivatives of the multivariate finit time ruin probability with respect to the arrival intensity, $\lambda$, at zero can be expressed in terms of certain sets of functions, in general $\left\{H_{t, u, x, y}^{k}\right\}$, define recursively using double integrals.

As explained in Section 3, the double Laplace transform of the mentioned sets of functions $\left\{H_{t, u, x, y}^{k * *}\right\}$ can be easily obtained recursively using the results of Theorem 3 .

The use of (3.4) instead of (3.3) generates expressions of reduced complexity and leads us to a substantial save in the number of computations, as it is proved in Usabel (1999).

However, in case Invd $\left\{\mathcal{F}_{m, n, x, y}^{k * *} ;(t, u)\right\}$ is obtained numerically, using (3.4), the accuracy will be endangered for increasing values of $t$ and a growing number of terms in the Taylor expansion $(L)$.

In order to obtain the coefficient we have to use a Laplace transform inversion formula twice (see Remark 1) for $k=1,2, \ldots, L$. It is easy to see that many Laplace inversion techniques have the following general formula (see for instance Davies and Martin (1979)

$$
H_{t, u, x, y}^{k}=\operatorname{Inv}\left\{H_{m, u, x, y}^{k *} ; t\right\} \simeq \sum_{i=1}^{\rho} W_{i}(t) H_{M_{i}(t), u, x, y}^{k *},
$$

where

$$
H_{M_{i}(t), u, x, y}^{k *}=\operatorname{Inv}\left\{H_{M_{i}(t), n, x, y}^{k * *} ; u\right\} \simeq \sum_{j=1}^{v} Y_{j}(u) H_{M_{i}(t), N_{j}(u), x, y}^{k *} .
$$

For each particular value of $m$ necessary in the inversion technique $\left(M_{1}(t), M_{2}(t), \ldots, M_{\rho}(t)\right)$ we should obtain

$$
H_{M_{i}(t), N_{j}(u), x, y}^{k * *}, \quad j=1,2, \ldots, \nu .
$$

For $M_{i}(t) \neq N_{j}(u)$ the family of functions $H_{M_{i}(t), N_{j}(u), x, y}^{k *}, \quad k=1,2, \ldots, L$, can be easily obtained using recursive expression (2.2) and initial condition (2.3) of Theorem 3: 


\begin{tabular}{|c|c|c|c|c|c|c|}
\hline$Z_{1}^{4}\left(M_{i}(t)_{i}(t)\right)$ & $\Gamma$ & $Z_{2}^{4}\left(M_{i}(t)\right)$ & $\Gamma$ & $Z_{3}^{4}\left(M_{i}(t)\right)$ & $\Gamma$ & $Z_{4}^{4}\left(M_{i}(t)\right)$ \\
\hline$Z_{1}^{3}\left(M_{i}(t)_{i}(t)\right)$ & $\gg$ & $Z_{2}^{3}\left(M_{i}(t)\right)$ & $r$ & $Z_{3}^{3}\left(M_{i}(t)\right)$ & & \\
\hline$Z_{1}^{2}\left(M_{i}(t)_{i}(t)\right)$ & $r$ & $Z_{2}^{2}\left(M_{i}(t)\right)$ & & & & \\
\hline$Z_{1}^{1}\left(M_{i}(t)_{i}(t)\right)$ & & & & & & \\
\hline
\end{tabular}

Fig. 1.

$$
\begin{aligned}
H_{M_{i}(t), N_{j}(u), x, y}^{(k+1) *}= & \frac{H_{M_{i}(t), N_{j}(u), x, y}^{k * *}\left(b^{*}\left(N_{j}(u)\right)-1\right)-H_{M_{i}(t), M_{i}(t), x, y}^{k * *}\left(b^{*}\left(M_{i}(t)\right)-1\right)}{M_{i}(t)-N_{j}(u)} \\
& +\frac{D_{x, y}^{(k+1) *}\left(N_{j}(u)\right)-D_{x, y}^{(k+1) *}\left(M_{i}(t)\right)}{M_{i}(t)-N_{j}(u)} \quad H_{M_{i}(t), N_{j}(u), x, y}^{1 * *}=\frac{\left(D_{x, y}^{1 *}\left(N_{j}(u)\right)-D_{x, y}^{1 *}\left(M_{i}(t)\right)\right)}{M_{i}(t)-N_{j}(u)}
\end{aligned}
$$

(where $D_{x, y}^{k *}(s)=F_{x, y}^{*}(s)\left(g^{*}(s)\right)^{k-1}$, see (3.2)).

Obviously, in order to use the former expression, the following set of functions must be obtained firs using (2.4)

$$
H_{M_{i}(t), M_{i}(t), x, y}^{k * *}=\frac{Z_{k}^{k}\left(M_{i}(t)\right)}{(-1)^{m} k !}, \quad i=1,2, \ldots, \alpha
$$

(and they are also the case $M_{i}(t)=N_{j}(u)$ ).

Functions $Z_{k}^{k}\left(M_{i}(t)\right)$ are again easily obtained by recursion using expressions (2.5) and (2.6) of Theorem 3 :

$$
\begin{aligned}
Z_{l}^{j}\left(M_{i}(t)\right) & =\sum_{i=l-1}^{j}\left(\begin{array}{l}
j \\
i
\end{array}\right) Z_{l-1}^{i}\left(M_{i}(t)\right)\left(b^{*}\left(M_{i}(t)\right)-1\right)^{\prime(j-i)}+\left(\frac{(-1)^{l-1} j !}{(j-(l-1)) !}\right)\left(D_{x, y}^{l *}\left(M_{i}(t)\right)\right)^{\prime(j-(l-1))}, \\
l & =1,2,3, \ldots, \quad j=l, l+1, \ldots
\end{aligned}
$$

and

$$
Z_{1}^{j}\left(M_{i}(t)\right)=\left(D_{x, y}^{1 *}\left(M_{i}(t)\right)\right)^{\prime(j)}, \quad j=1,2, \ldots
$$

It is easy to prove, from the former recursive formula, that in order to get $Z_{l}^{j}\left(M_{i}(t)\right)$ we need to obtain firs $Z_{l-1}^{l-1}\left(M_{i}(t)\right), Z_{l-1}^{l}\left(M_{i}(t)\right), \ldots, Z_{l-1}^{j}\left(M_{i}(t)\right)$. This fact is showed graphically in Fig 1.

As a consequence, the total number of functions $Z_{l}^{j}\left(M_{i}(t)\right)$ to evaluate in order to obtain $Z_{L}^{L}\left(M_{i}(t)\right), i=$ $1,2, \ldots \rho$, will be $(L+1) L / 2$, in which $Z_{k}^{k}\left(M_{i}(t)\right), k=1, \ldots, L-1$, are also included.

Moreover, it is not hard to prove either that the total number of functions $H_{M_{i}(t), N_{j}(u), x, y}^{k * *}$ and $Z_{k}^{j}\left(M_{i}(t)\right)$ to evaluate with the former easy expressions of Theorem 3 are

$$
\rho \times v \times L+\rho \times \frac{(L+1) L}{2}
$$

With the former number of calculations we will be able to approximate all the coefficient of the Taylor series expansion of order $L(4.1)$.

In summary, using the derivatives

$$
\begin{aligned}
& \left(D_{x, y}^{k *}(s)\right)^{\prime(j)}=\left(F_{x, y}^{*}(s)\left(g^{*}(s)\right)^{k-1}\right)^{\prime(j)}, \quad k=1, \ldots, L, j=0, \ldots, L-k, \\
& \left(b^{*}(s)-1\right)^{\prime(i)}, \quad i=0, \ldots, L,
\end{aligned}
$$


where functions $F_{x, y}^{*}(s), g^{*}(s)$ and $b^{*}(s)$ are previously known and the easy recursive formulas stated in Theorem 3 , we will be able to obtain the family of functions

$$
\left\{\Xi_{t, u, x, y}^{k * *}\right\}, \quad\left\{\mathcal{F}_{t, u, x, y}^{k * *}\right\}, \quad k=1,2,3, \ldots,
$$

and finall fin the coefficient of the Taylor expansion with formulas (3.3) or (3.4) respectively, inverting Laplace transforms (single and double).

\section{Efficienc of the algorithm}

Once the theoretical foundations of this approach has been settled down and a practical algorithm has been also produced, designed to calculate the coefficient of the Taylor series, the attention will be now focused on the efficien $y$.

Efficien y will be clearly based upon the figur of the total number of calculations obtained in the former Section 4.4, in order to get approximations of the coefficient of the truncated Taylor series expansion (4.1).

It is important to mention that this total number of calculations does not depend at all on the magnitude of the initial reserves considered or time span. Moreover, the recursions formulas introduced in Theorem 3 are so easy that the evaluation of the former total number of calculations will be attained extremely fast.

The firs important issue to care about is the efficien y of methods of inverting Laplace transforms because parameters $\rho$ and $v$ will be affected by this fact. This aspect of numerical calculus is beyond the scope of this work but we can certainly state that scientifi literature and computer languages libraries can provide us with efficien tools in order to solve this problem. One of the most cited papers regarding comparison of numerical methods of inverting Laplace transform is Davies and Martin (1979). In the numerical examples presented, using Gaver-Stehfest method, Gaver (1966) and Stehfest (1970), with $\rho=14$ and $v=30$, results yielded 4-5 significan digits.

We should mention that the use of numerical methods of inverting Laplace transform is not new at all in actuarial literature. As we stated in the last paragraph of Section 1, many classical works of the 1970s Scandinavian School (and H. Seal) were based on the inversion of double Laplace transforms as well. The results obtained by these methods have been extensively cited later and used as the "true value" of ruin probabilities to compare with the many approximations based on discretizations produced in the 1980s.

A fina additional consideration about the efficien $y$, also beyond the scope of this work but certainly an interesting area of future research, is the number of terms of the truncated Taylor series expansion (4.1) in order to get a desired degree of accuracy when approximating multivariate finit time ruin probabilities for the classical case of risk theory. We will see later that in the numerical illustration considered, the truncation sum limit $\mathrm{L}$ should be increased for larger time horizons considered in order to guarantee a certain accuracy.

\section{Numerical illustration}

Using basic properties of Laplace transforms,

$$
\begin{aligned}
L\left(s, F_{x, y}^{1}(u)\right)= & \int_{0}^{x} \mathrm{e}^{-s u} \int_{u}^{x}(g(z)-g(y+z)) \mathrm{d} z \mathrm{~d} u=\left(\frac{1}{s}\right) \int_{0}^{x}(g(z)-g(y+z)) \mathrm{d} z \\
& -\left(\frac{1}{s}\right) \int_{0}^{x}(g(z)-g(y+z)) \mathrm{e}^{-s z} \mathrm{~d} z \\
L\left(s, F_{x, y}^{2}(u)\right)= & \int_{0}^{\infty} \mathrm{e}^{-s u} \int_{u}^{u+x}(g(z)-g(y+z)) \mathrm{d} z \mathrm{~d} u=\frac{L\left(s, F_{x, y}^{\prime 2}(u)\right)+F_{x, y}^{2}(0)}{s}
\end{aligned}
$$

where 


$$
F_{x, y}^{2}(0)=\int_{0}^{x}(g(z)-g(y+z)) \mathrm{d} z, \quad x>0,
$$

and Leibniz's differentiation theorem leads to

$$
F_{x, y}^{\prime 2}(u)=g(u+x)-g(u+x+y)+g(u+y)-g(u)
$$

and

$$
L\left(s, F_{x, y}^{\prime 2}(u)\right)=L(s, g(u+x))-L(s, g(u+x+y))+L(s, g(u+y))-L(s, g(u)),
$$

or

$$
\begin{aligned}
L(s, g(u+a)) & =\int_{0}^{\infty} \mathrm{e}^{-s u} g(u+a) \mathrm{d} u=\int_{0}^{\infty} \mathrm{e}^{-s u}(1-B(u+a)) \mathrm{d} u=\frac{1}{s}-L(s, B(u+a)) \\
& =\frac{1-B(a)-L(s, b(u+a))}{s} .
\end{aligned}
$$

As an example we will consider Pareto claim size distribution

$$
B(x)=1-\left(\frac{\alpha}{\alpha+x}\right)^{\alpha+1}, \quad x>0, \quad \alpha \geq 1, \quad E_{b}(x)=\mu^{-1}=1
$$

and

$$
g(x)=\left(\frac{\alpha}{\alpha+x}\right)^{\alpha+1}, \quad x>0 .
$$

Definin the integral following expressions (3.353.1) and (3.353.2) of Gradshteyn and Ryzhik (1994):

$$
\begin{aligned}
\varphi(\sigma, \beta, n, \gamma)= & \int_{0}^{\gamma} \frac{\mathrm{e}^{-\sigma x} \mathrm{~d} x}{(x+\beta)^{n}}=\frac{1}{(n-1) !} \sum_{k=1}^{n-1}(k-1) !(-\sigma)^{n-1-k}\left(\beta^{-k}-\frac{\mathrm{e}^{-\gamma \sigma}}{(\gamma+\beta)^{k}}\right) \\
& -\frac{(-\sigma)^{n-1} \mathrm{e}^{\beta \sigma}}{(n-1) !}(\operatorname{Ei}(-\beta \sigma)-\operatorname{Ei}(-(\gamma+\beta) \sigma)), \quad n \geq 2, \quad \operatorname{Re} \sigma>0, \quad|\arg (\gamma+\beta)|<\pi,
\end{aligned}
$$

where Ei is the exponential integral

$$
\operatorname{Ei}(x)=-\int_{1}^{\infty} \frac{\mathrm{e}^{x t}}{t} \mathrm{~d} t, \quad x<0
$$

and as a particular case

$$
\varphi(\sigma, \beta, n,+\infty)=\frac{1}{(n-1) !} \sum_{k=1}^{n-1}(k-1) !(-\sigma)^{n-1-k} \beta^{-k}-\frac{(-\sigma)^{n-1} \mathrm{e}^{\beta \sigma}}{(n-1) !} \operatorname{Ei}(-\beta \sigma) .
$$

It is easy to prove that

$$
\begin{aligned}
& b^{*}(s)=\alpha^{\alpha+1}(\alpha+1) \varphi(s, \alpha, \alpha+2,+\infty) \\
& g^{*}(s)=\alpha^{\alpha+1} \varphi(s, \alpha, \alpha+1,+\infty), \quad s>0,
\end{aligned}
$$


finall

$$
\begin{aligned}
L\left(s, F_{x, y}^{1}(u)\right)= & \left(\frac{1}{s}\right) \int_{0}^{x}(g(z)-g(y+z)) \mathrm{d} z-\left(\frac{1}{s}\right) \int_{0}^{x}(g(z)-g(y+z)) \mathrm{e}^{-s z} \mathrm{~d} z \\
= & \alpha^{\alpha+1} \frac{\alpha^{-\alpha}-(\alpha+x)^{-\alpha}-(\alpha+y)^{-\alpha}+(\alpha+y+x)^{-\alpha}}{s \alpha} \\
& -\alpha^{\alpha+1} \frac{\varphi(s, \alpha, \alpha+1, x)-\varphi(s, \alpha+y, \alpha+1, x)}{s}
\end{aligned}
$$

and

$$
\begin{aligned}
L\left(s, F_{x, y}^{2}(u)\right)= & \int_{0}^{\infty} \mathrm{e}^{-s u} \int_{u}^{u+x}(g(z)-g(y+z)) \mathrm{d} z \mathrm{~d} u=\left(\frac{1}{s}\right) L(s, g(u+x))-\left(\frac{1}{s}\right) L(s, g(u+x+y)) \\
& +\left(\frac{1}{s}\right) L(s, g(u+y))-\left(\frac{1}{s}\right) L(s, g(u))+\left(\frac{1}{s}\right) F_{x, y}^{2}(0) \\
= & \alpha^{\alpha+1} \frac{\varphi(s, \alpha+x, \alpha+1,+\infty)-\varphi(s, \alpha+y+x, \alpha+1,+\infty)}{s} \\
& +\alpha^{\alpha+1} \frac{\varphi(s, \alpha+y, \alpha+1,+\infty)-\varphi(s, \alpha, \alpha+1,+\infty)}{s} \\
& +\alpha^{\alpha+1} \frac{\alpha^{-\alpha}-(\alpha+x)^{-\alpha}-(\alpha+y)^{-\alpha}+(\alpha+y+x)^{-\alpha}}{s \alpha}
\end{aligned}
$$

As a numerical illustration we have obtained Maclaurin's truncated approximations up to eight terms using the algorithm presented in Section 4

$$
\Psi_{t, u, 20,1}(\lambda) \simeq \Psi_{t, u, 20,1}^{L}(\lambda)=\sum_{k=1}^{L} \frac{\Psi_{t, u, 20,1}^{(k)}(0)}{k !} \lambda^{k}, \quad L=1,2, \ldots, 8
$$

for the following set of parameters:

$$
\begin{aligned}
& \alpha=1, \quad y=1, \quad x=20, \quad F_{20,1}^{2}(w), \quad X=Z_{\tau^{-}}=u+20 \text { (surplus prior to ruin), } \\
& u=10,20, \quad t=5,10,20 .
\end{aligned}
$$

Numerical inversion of double Laplace transform was performed using Gaver-Stehfest method twice with the suitable choice of parameters (usually $\rho=14$ and $v=30$, see (4.2) and (4.3)) in order to guarantee at least 4-5 significan digits accuracy; see for instance Davies and Martin (1979) or Stehfest (1970).

The coefficient of the Maclaurin's polynomials of order eight with respect to the arrival intensity were obtained for $u=10$ :

$$
\begin{aligned}
\Psi_{5,10,20,1}^{8}(\lambda)= & 0.003642 \lambda+0.004123 \lambda^{2}+0.002761 \lambda^{3}+0.001037+0.00009681 \lambda^{5}-0.00007615 \lambda^{6} \\
- & 0.00002213 \lambda^{7}-0.160710^{-5} \lambda^{8}, \\
\Psi_{10,10,20,1}^{8}(\lambda)= & 0.004984 \lambda+0.007464 \lambda^{2}+0.007282 \lambda^{3}+0.004823 \lambda^{4}+0.001762 \lambda^{5}-0.00017121 \lambda^{6} \\
& -0.0005476 \lambda^{7}-0.00017790 \lambda^{8}, \\
\Psi_{20,10,20,1}^{8}(\lambda)= & 0.005937 \lambda+0.01057 \lambda^{2}+0.01305 \lambda^{3}+0.01228 \lambda^{4}+0.008459 \lambda^{5}+0.003254 \lambda^{6} \\
& -0.0009310 \lambda^{7}-0.002488 \lambda^{8},
\end{aligned}
$$

and for $u=20$, 
Table 1

Approximations for multivariate finit time ruin probability for Pareto distribution claim size $(6.4), \alpha=1, u=10, t=5,10,20, \Psi_{t, 10,20,1}^{L}(\lambda)=$ $\sum_{k=1}^{L} \frac{\Psi_{t, 10,20,1}^{\prime(k)}}{k !} \lambda^{k},(-n) \equiv 10^{-n}, \rightarrow$ same as the previous figur

\begin{tabular}{|c|c|c|c|c|c|c|c|c|c|}
\hline & \multirow[t]{2}{*}{$\lambda$} & \multicolumn{8}{|l|}{$L$} \\
\hline & & 1 & 2 & 3 & 4 & 5 & 6 & 7 & 8 \\
\hline \multirow[t]{5}{*}{$t=5$} & 0.10 & $3.642(-4)$ & $4.054(-4)$ & $4.082(-4)$ & $4.083(-4)$ & $\rightarrow$ & $\rightarrow$ & $\rightarrow$ & $\rightarrow$ \\
\hline & 0.25 & $9.105(-4)$ & $1.168(-3)$ & $1.211(-3)$ & $1.215(-3)$ & $\rightarrow$ & $\rightarrow$ & $\rightarrow$ & $\rightarrow$ \\
\hline & 0.50 & $1.821(-3)$ & $2.852(-3)$ & $3.197(-3)$ & $3.261(-3)$ & $3.265(-3)$ & $3.263(-3)$ & $\rightarrow$ & $\rightarrow$ \\
\hline & 0.75 & $2.732(-3)$ & $5.051(-3)$ & $6.215(-3)$ & $6.543(-3)$ & $6.566(-3)$ & $6.553(-3)$ & $6.550(-3)$ & $\rightarrow$ \\
\hline & 1.00 & $3.642(-3)$ & $7.765(-3)$ & $1.053(-2)$ & $1.156(-2)$ & $1.166(-2)$ & $1.158(-2)$ & $1.156(-2)$ & $\rightarrow$ \\
\hline \multirow[t]{5}{*}{$t=10$} & 0.10 & $4.984(-4)$ & $5.730(-4)$ & $5.803(-4)$ & $5.508(-4)$ & $\rightarrow$ & $\rightarrow$ & $\rightarrow$ & $\rightarrow$ \\
\hline & 0.25 & $1.246(-3)$ & $1.713(-3)$ & $1.827(-3)$ & $1.846(-3)$ & $1.848(-3)$ & $\rightarrow$ & $\rightarrow$ & $\rightarrow$ \\
\hline & 0.50 & $2.492(-3)$ & $4.358(-3)$ & $5.268(-3)$ & $5.569(-3)$ & $5.624(-3)$ & $5.621(-3)$ & $5.617(-3)$ & $5.616(-3)$ \\
\hline & 0.75 & $3.738(-3)$ & $7.937(-3)$ & $1.101(-2)$ & $1.254(-2)$ & $1.296(-2)$ & $1.293(-2)$ & $1.286(-2)$ & $1.284(-2)$ \\
\hline & 1.00 & $4.984(-3)$ & $1.245(-2)$ & $1.973(-2)$ & $2.455(-2)$ & $2.631(-2)$ & $2.614(-2)$ & $2.559(-2)$ & $2.541(-2)$ \\
\hline \multirow[t]{5}{*}{$t=20$} & 0.10 & $5.937(-4)$ & $6.994(-4)$ & $7.125(-4)$ & $7.137(-4)$ & $7.138(-4)$ & $\rightarrow$ & $\rightarrow$ & $\rightarrow$ \\
\hline & 0.25 & $1.484(-3)$ & $2.145(-3)$ & $2.349(-3)$ & $2.397(-3)$ & $2.405(-3)$ & $2.406(-3)$ & $\rightarrow$ & $\rightarrow$ \\
\hline & 0.50 & $2.969(-3)$ & $5.162(-3)$ & $7.243(-3)$ & $8.011(-3)$ & $8.275(-3)$ & $8.326(-3)$ & $8.319(-3)$ & $8.309(-3)$ \\
\hline & 0.75 & $4.453(-3)$ & $1.040(-2)$ & $1.591(-2)$ & $1.980(-2)$ & $2.181(-2)$ & $2.239(-2)$ & $2.227(-2)$ & $2.202(-2)$ \\
\hline & 1.00 & $5.937(-3)$ & $1.651(-2)$ & $2.956(-2)$ & $4.185(-2)$ & $5.031(-2)$ & $5.357(-2)$ & $5.264(-2)$ & $5.015(-2)$ \\
\hline
\end{tabular}

$$
\begin{aligned}
\Psi_{5,20,20,1}^{8}(\lambda)= & 0.0006219 \lambda+0.0006242 \lambda^{2}+0.0003745 \lambda^{3}+0.0001362 \lambda^{4}+0.00002404 \lambda^{5} \\
& +0.1271 \times 10^{-5} \lambda^{6}+0.9100 \times 10^{-6} \lambda^{7}+0.3551 \times 10^{-6} \lambda^{8} \\
\Psi_{10,20,20,1}^{8}(\lambda)= & 0.0009528 \lambda+0.001323 \lambda^{2}+0.001202 \lambda^{3}+0.0007883 \lambda^{4}+0.0003479 \lambda^{5}+0.00007517 \lambda^{6} \\
& -0.6173 \times 10^{-5} \lambda^{7}-0.7448 \times 10^{-5} \lambda^{8}, \\
\Psi_{20,20,20,1}^{8}(\lambda)= & 0.001267 \lambda+0.002226 \lambda^{2}+0.002694 \lambda^{3}+0.002590 \lambda^{4}+0.001988 \lambda^{5}+0.001139 \lambda^{6} \\
& +0.0003723 \lambda^{7}-0.00006635 \lambda^{8} .
\end{aligned}
$$

We can easily deduce from the polynomial approximations presented above that the coefficient of the last terms of the sums, $\Psi_{t, u, 20,1}^{\prime(8)}(0) / 8$ ! (as a measure of the truncation error), increase with time; in other words the accuracy obtained with Maclaurin's expansions of order 8 worsen as longer time spans $(t)$ are considered (see Tables 1 and 2). Obviously, the desired level of accuracy will be reached using Maclaurin's expansions of higher order.

The figure for the coefficient of the polynomials above were obtained using a simple algorithm in Maple $\mathrm{V}$ Release 4 on a Pentium 120. The computation time for the eight coefficient (all of them) of any of the polynomials ranged from 20 to $40 \mathrm{~s}$. Bearing in mind that a program as Maple $\mathrm{V}$ performs operations 50-500 times slower than languages as FORTRAN or C (see Char et al., 1991, p. 84), the same algorithm takes fractions of a second in one of these languages. We can then conclude that the efficien y of the algorithm in terms of computation time is also outstanding.

\section{Concluding comments}

In the present work we have enhanced the practical application of power series expansion with respect to the arrival intensity $\lambda$ in order to approximate multivariate finit time ruin probabilities. Using double Laplace transforms, the complicated recursive formulas based on double integrals, (1.4) and (1.9), were transformed, respectively, into very easy recursive formulas presented in Theorem 3. The algorithm presented in Section 4 can then be considered as efficien in terms of computational times as it is explained in Sections 5 and 6. 
Table 2

Approximations for multivariate finit time ruin probability for Pareto distribution claim size $(6.4), \alpha=1, u=10, t=5,10,20, \Psi_{t, 20,20,1}^{L}(\lambda)=$ $\sum_{k=1}^{L} \frac{\Psi_{t, 20,20,1}^{\prime(k)}}{k !} \lambda^{k},(-n) \equiv 10^{-n}, \rightarrow$ same as the previous figur

\begin{tabular}{|c|c|c|c|c|c|c|c|c|c|}
\hline & \multirow[t]{2}{*}{$\lambda$} & \multicolumn{8}{|l|}{$\mathrm{L}$} \\
\hline & & 1 & 2 & 3 & 4 & 5 & 6 & 7 & 8 \\
\hline \multirow[t]{5}{*}{$t=5$} & 0.10 & $6.219(-5)$ & $6.843(-5)$ & $6.880(-5)$ & $6.881(-5)$ & $\rightarrow$ & $\rightarrow$ & $\rightarrow$ & $\rightarrow$ \\
\hline & 0.25 & $1.555(-4)$ & $1.945(-4)$ & $2.004(-4)$ & $2.009(-4)$ & $\rightarrow$ & $\rightarrow$ & $\rightarrow$ & $\rightarrow$ \\
\hline & 0.50 & $3.110(-4)$ & $4.671(-4)$ & $5.139(-4)$ & $5.224(-4)$ & $5.232(-4)$ & $\rightarrow$ & $\rightarrow$ & $\rightarrow$ \\
\hline & 0.75 & $4.664(-4)$ & $8.175(-4)$ & $9.755(-4)$ & $1.019(-3)$ & $1.025(-3)$ & $\rightarrow$ & $\rightarrow$ & $\rightarrow$ \\
\hline & 1.00 & $6.219(-4)$ & $1.246(-3)$ & $1.621(-3)$ & $1.757(-3)$ & $1.781(-3)$ & $1.782(-3)$ & $1.783(-3)$ & $\rightarrow$ \\
\hline \multirow[t]{5}{*}{$t=10$} & 0.10 & $9.528(-5)$ & $1.085(-4)$ & $1.097(-4)$ & $1.098(-4)$ & $\rightarrow$ & $\rightarrow$ & $\rightarrow$ & $\rightarrow$ \\
\hline & 0.25 & $2.382(-4)$ & $3.209(-4)$ & $3.397(-4)$ & $3.428(-4)$ & $3.431(-4)$ & $\rightarrow$ & $\rightarrow$ & $\rightarrow$ \\
\hline & 0.50 & $4.764(-4)$ & $8.072(-4)$ & $9.575(-4)$ & $1.007(-3)$ & $1.018(-3)$ & $1.019(-3)$ & $\rightarrow$ & $\rightarrow$ \\
\hline & 0.75 & $7.146(-4)$ & $1.459(-3)$ & $1.966(-3)$ & $2.215(-3)$ & $2.298(-3)$ & $2.311(-3)$ & $2.310(-3)$ & $2.309(-3)$ \\
\hline & 1.00 & $9.528(-4)$ & $2.276(-3)$ & $3.478(-3)$ & $4.266(-3)$ & $4.614(-3)$ & $4.689(-3)$ & $4.683(-3)$ & $4.676(-3)$ \\
\hline \multirow[t]{5}{*}{$t=20$} & 0.10 & $1.268(-4)$ & $1.491(-4)$ & $1.518(-4)$ & $1.521(-4)$ & $\rightarrow$ & $\rightarrow$ & $\rightarrow$ & $\rightarrow$ \\
\hline & 0.25 & $3.170(-4)$ & $4.561(-4)$ & $4.982(-4)$ & $5.083(-4)$ & $5.102(-4)$ & $5.105(-4)$ & $\rightarrow$ & $\rightarrow$ \\
\hline & 0.50 & $6.340(-4)$ & $1.191(-3)$ & $1.528(-3)$ & $1.690(-3)$ & $1.752(-3)$ & $1.770(-3)$ & $1.773(-3)$ & $\rightarrow$ \\
\hline & 0.75 & $9.510(-4)$ & $2.203(-3)$ & $3.340(-3)$ & $4.160(-3)$ & $4.632(-3)$ & $4.835(-3)$ & $4.885(-3)$ & $4.887(-3)$ \\
\hline & 1.00 & $1.268(-3)$ & $3.494(-3)$ & $6.188(-3)$ & $8.778(-3)$ & $1.077(-2)$ & $1.191(-2)$ & $1.228(-2)$ & $1.221(-3)$ \\
\hline
\end{tabular}

In the example presented in Section 6, the degree of Maclaurin's polynomials in order to guarantee a certain desired accuracy is increasing with the time horizon $(t)$ considered. It is easy to conclude, then, that power series expansion will work particularly well for not large time spans because the number of terms necessary to obtain a desired accuracy will be small.

This last fact is most important because, citing Frey and Schmidt (1996), other works in actuarial literature based on diffusion approximations: Asmussen (1984), Grandell (1977), Igehart (1969), or Schmidli (1992) gave reasonable accuracy for large time horizons because they were based on a limit theorem; and the Maclaurin's polynomial expansions may be the complement for not large time horizons.

\section{Appendix A. Proof of Theorem 3}

Let us start the proof with this simple lemma.

Lemma 1. The double Laplace transform of a general function $D_{x, y}(u+t)$ can be expressed as

$$
L\left(D_{x, y}(u+t) ;(m, n)\right)= \begin{cases}\frac{D_{x, y}^{*}(n)-D_{x, y}^{*}(m)}{m-n}, & n \neq m, \\ -\mathrm{d} D_{x, y}^{*}(m) / \mathrm{d} m=\left(D_{x, y}^{*}(m)\right)^{\prime(1)}, & n=m .\end{cases}
$$

\section{Proof.}

$$
\begin{aligned}
L\left(D_{x, y}(u+t) ;(m, n)\right)= & \int_{t=0}^{\infty} \int_{u=0}^{\infty} D_{x, y}(u+t) \mathrm{e}^{-m t-n u} \mathrm{~d} u \mathrm{~d} t=\int_{z=0}^{\infty} \int_{t=0}^{z} D_{x, y}(z) \mathrm{e}^{-m t-(z-t) n} \mathrm{~d} z \mathrm{~d} t \\
& =\int_{z=0}^{\infty} D_{x, y}(z) \mathrm{e}^{-n z} \int_{t=0}^{z} \mathrm{e}^{-(m-n) t} \mathrm{~d} t \mathrm{~d} z=D_{m, n, x, y}^{* *}=\frac{D_{x, y}^{*}(n)-D_{x, y}^{*}(m)}{m-n}
\end{aligned}
$$

with the change of variable

$$
u+t=z, \quad t=t
$$


The formula for the special case $n=m$ is easily obtained using the definitio of derivative of a real function.

Expression (2.3) of Theorem 3 is then obtained using Lemma 1 in (2.1).

With the aid of the following lemma, expression (2.2) of Theorem 3 will be proved and an interesting result is also obtained for the fina proof.

Lemma 2. Let us consider a certain family of functions, $D_{x, y}^{k}(z)(k=1,2, \ldots)$ and $b(z)$, with infinitel differentiable Laplace transforms.

If a set of functions $H_{t, u, x, y}^{k}(k=1,2, \ldots)$ can be obtained using the mentioned family and the following recursive expression

$$
\begin{aligned}
H_{t, u, x, y}^{k+1} & =\int_{s=0}^{t} \int_{z=0}^{u+s} H_{t-s, u+s-z, x, y}^{k} b(z) \mathrm{d} z \mathrm{~d} s-\int_{s=0}^{t} H_{t-s, u+s, x, y}^{k} \mathrm{~d} s+D_{x, y}^{k+1}(u+t) \\
& =\mathcal{I}_{1}\left(H_{t, u, x, y}^{k}\right)-\mathcal{I}_{2}\left(H_{t, u, x, y}^{k}\right)+D_{x, y}^{k+1}(u+t)
\end{aligned}
$$

with initial condition

$$
H_{t, u, x, y}^{1}=D_{x, y}^{1}(u+t)
$$

then the double Laplace transform of this set can be expressed as

$$
H_{m, n, x, y}^{(k+1) * *}=\frac{H_{m, n, x, y}^{k * *}\left(b^{*}(n)-1\right)-H_{m, m, x, y}^{k * *}\left(b^{*}(m)-1\right)}{m-n}+\frac{\left(D_{x, y}^{(k+1) *}(n)-D_{x, y}^{(k+1) *}(m)\right)}{m-n}
$$

with initial condition

$$
H_{m, n, x, y}^{1 * *}=\frac{\left(D_{x, y}^{1 *}(n)-D_{x, y}^{1 *}(m)\right)}{m-n}
$$

or

$$
H_{m, n, x, y}^{(k+1) * *}= \begin{cases}R H_{m, n, x, y}^{(k+1) * *} /(m-n)^{k+1}, & n \neq m, \\ \left(\partial^{k+1}\left(R H_{m, n, x, y}^{(k+1) * *}\right)_{n=m} / \partial n^{k+1}\right)\left((-1)^{k+1}(k+1) !\right)^{-1}, & n=m,\end{cases}
$$

where functions $R H_{m, n, x, y}^{(k+1) * *}$ are define also recursively

$$
\begin{aligned}
R H_{m, n, x, y}^{(k+1) * *}= & R H_{m, n, x, y}^{(k) * *}\left(b^{*}(n)-1\right)-H_{m, m, x, y}^{(k) * *}\left(b^{*}(m)-1\right)(m-n)^{k}+(m-n)^{k}\left(D_{x, y}^{(k+1) *}(n)\right. \\
& -D_{x, y}^{(k+1) *(m))}
\end{aligned}
$$

with initial condition

$$
R H_{m, n, x, y}^{1 * *}=D_{x, y}^{1 *}(n)-D_{x, y}^{1 *}(m) .
$$

Proof. Let us prove firs the following recursive expression:

$$
H_{m, n, x, y}^{(k+1) * *}=\frac{H_{m, n, x, y}^{k * *}\left(b^{*}(n)-1\right)-H_{m, m, x, y}^{k * *}\left(b^{*}(m)-1\right)}{m-n}+\frac{\left(D_{x, y}^{(k+1) *}(n)-D_{x, y}^{(k+1) *}(m)\right)}{m-n} .
$$


Using the definitio of the double Laplace transform

$$
\begin{aligned}
L\left(\mathcal{I}_{1}\left(H_{t, u, x, y}^{k}\right) ;(m, n)\right)= & \int_{t=0}^{\infty} \int_{u=0}^{\infty} \int_{s=0}^{t} \int_{z=0}^{u+s} H_{t-s, u+s-z, x, y}^{k} b(z) \mathrm{d} z \mathrm{~d} s \mathrm{e}^{-m t-n u} \mathrm{~d} u \mathrm{~d} t \\
= & \int_{s=0}^{\infty} \int_{z=0}^{\infty}\left(\int_{a=0}^{\infty} \int_{b=(s-z)^{+}}^{\infty} H_{a, b, x, y}^{k} b(z) \mathrm{e}^{-(m-n) s-n z} \mathrm{~d} z \mathrm{~d} s\right) \mathrm{e}^{-m a-n b} \mathrm{~d} a \mathrm{~d} b \\
= & \int_{s=0}^{\infty} \int_{z=s}^{\infty}\left(\int_{a=0}^{\infty} \int_{b=0}^{\infty} H_{a, b, x, y}^{k} b(z) \mathrm{e}^{-(m-n) s-n z} \mathrm{~d} z \mathrm{~d} s\right) \mathrm{e}^{-m a-n b} \mathrm{~d} a \mathrm{~d} b \\
& +\int_{s=0}^{\infty} \int_{z=0}^{s}\left(\int_{a=0}^{\infty} \int_{b=(s-z)}^{\infty} H_{a, b, x, y}^{k} b(z) \mathrm{e}^{-(m-n) s-n z} \mathrm{~d} z \mathrm{~d} s\right) \mathrm{e}^{-m a-n b} \mathrm{~d} a \mathrm{~d} b \\
= & \int_{a=0}^{\infty} \int_{b=0}^{\infty} H_{a, b, x, y}^{k} \mathrm{e}^{-m a-n b} \int_{z=0}^{\infty} b(z) \mathrm{e}^{-n z} \int_{s=0}^{z} \mathrm{e}^{-(m-n) s} \mathrm{~d} s \mathrm{~d} z \mathrm{~d} b \mathrm{~d} a \\
& +\int_{a=0}^{\infty} \int_{b=0}^{\infty} H_{a, b, x, y}^{k} \mathrm{e}^{-m a-n b} \int_{z=0}^{\infty} b(z) \mathrm{e}^{-n z} \int_{s=z}^{b+z} \mathrm{e}^{-(m-n) s} \mathrm{~d} s \mathrm{~d} z \mathrm{~d} b \mathrm{~d} a \\
= & \int_{a=0}^{\infty} \int_{b=0}^{\infty} H_{a, b, x, y}^{k} \mathrm{e}^{-m a-n b} \int_{z=0}^{\infty} b(z) \mathrm{e}^{-n z} \int_{s=0}^{b+z} \mathrm{e}^{-(m-n) s} \mathrm{~d} s \mathrm{~d} z \mathrm{~d} b \mathrm{~d} a \\
= & \frac{1}{m-n} \int_{a=0}^{\infty} \int_{b=0}^{\infty} H_{a, b, x, y}^{k} \mathrm{e}^{-m a-n b} \int_{z=0}^{\infty} b(z) \mathrm{e}^{-n z}\left(1-\mathrm{e}^{-(m-n)(b+z)}\right) \mathrm{d} z \mathrm{~d} b \mathrm{~d} a \\
= & \frac{H_{m, n, x, y}^{k * *} b^{*}(n)-H_{m, m, x, y}^{k * *} b^{*}(m)}{m-n}
\end{aligned}
$$

with the change of variables:

$$
t-s=a, \quad u+s-z=b, \quad s=s, \quad z=z,
$$

and

$$
\begin{aligned}
L\left(\mathcal{I}_{2}\left(H_{t, u, x, y}^{k}\right) ;(m, n)\right) & =\int_{t=0}^{\infty} \int_{u=0}^{\infty} \int_{s=0}^{t} H_{t-s, u+s, x, y}^{k} \mathrm{~d} s \mathrm{e}^{-m t-n u} \mathrm{~d} u \mathrm{~d} t \\
& =\int_{s=0}^{\infty} \int_{a=0}^{\infty} \int_{b=s}^{\infty} H_{a, b, x, y}^{k} \mathrm{e}^{-(m-n) s} \mathrm{~d} s \mathrm{e}^{-m a-n b} \mathrm{~d} a \mathrm{~d} b \\
& =\int_{a=0}^{\infty} \int_{b=0}^{\infty} H_{a, b, x, y}^{k} \mathrm{e}^{-m a-n b} \int_{s=0}^{b} \mathrm{e}^{-(m-n) s} \mathrm{~d} s \mathrm{~d} b \mathrm{~d} a \\
& =\frac{1}{m-n} \int_{a=0}^{\infty} \int_{b=0}^{\infty} H_{a, b, x, y}^{k} \mathrm{e}^{-m a-n b}\left(1-\mathrm{e}^{-(m-n) b}\right) \mathrm{d} b \mathrm{~d} a=\frac{H_{m, n, x, y}^{k * *}-H_{m, m, x, y}^{k * *}}{m-n}
\end{aligned}
$$

with the change of variables:

$$
t-s=a, \quad u+s=b, \quad s=s .
$$

Finally,

$$
\begin{aligned}
L\left(H_{t, u, x, y}^{k+1} ;(m, n)\right) & =H_{m, n, x, y}^{(k+1) * *}=L\left(\mathcal{I}_{1}\left(H_{t, u, x, y}^{k}\right) ;(m, n)\right)-L\left(\mathcal{I}_{2}\left(H_{t, u, x, y}^{k}\right) ;(m, n)\right) \\
& =\frac{H_{m, n, x, y}^{k * *}\left(b^{*}(n)-1\right)-H_{m, m, x, y}^{k * *}\left(b^{*}(m)-1\right)}{m-n}+\frac{\left(D_{x, y}^{(k+1) *}(n)-D_{x, y}^{(k+1) *}(m)\right)}{m-n}
\end{aligned}
$$


and

$$
H_{m, n, x, y}^{1 * *}=\frac{\left(D_{x, y}^{1 *}(n)-D_{x, y}^{1 *}(m)\right)}{m-n}
$$

using Lemma 1 for the last term and the initial function.

Let us now suppose that

$$
H_{m, n, x, y}^{(k) * *}=\frac{R H_{m, n, x, y}^{(k) * *}}{(m-n)^{k}}, \quad n \neq m,
$$

where $R H_{m, n, x, y}^{(k) * *}$ is define recursively with expression (A.2).

Proceeding by complete induction with (A.5):

$$
\begin{aligned}
H_{m, n, x, y}^{(k+1) * *} & =\frac{H_{m, n, x, y}^{k * *}\left(b^{*}(n)-1\right)-H_{m, m, x, y}^{k * *}\left(b^{*}(m)-1\right)}{m-n}+\frac{\left(D_{x, y}^{(k+1) *}(n)-D_{x, y}^{(k+1) *}(m)\right)}{m-n} \\
& =\left(\frac{\left.R H_{m, n, x, y}^{(k) * *} /(m-n)^{k}\right)\left(b^{*}(n)-1\right)-H_{m, m, x, y}^{k * *}\left(b^{*}(m)-1\right)}{m-n}+\frac{\left(D_{x, y}^{(k+1) *}(n)-D_{x, y}^{(k+1) *}(m)\right)}{m-n}\right. \\
& =\frac{R H_{m, n, x, y}^{(k) * *}\left(b^{*}(n)-1\right)-H_{m, m, x, y}^{k * *}\left(b^{*}(m)-1\right)(m-n)^{k}}{(m-n)^{k+1}}+\frac{(m-n)^{k}\left(D_{x, y}^{(k+1) *}(n)-D_{x, y}^{(k+1) *}(m)\right)}{(m-n)^{k+1}} \\
& =\frac{R H_{m, n, x, y}^{(k+1) * *}}{(m-n)^{k+1}},
\end{aligned}
$$

and obviously, using L'Hospital's rule,

$$
\lim _{n \rightarrow m} H_{m, n, x, y}^{(k+1) * *}=\frac{\partial^{k+1}\left(R H_{m, n, x, y}^{(k+1) * *}\right)_{n=m}}{\partial n^{k+1}(-1)^{k+1}(k+1) !}
$$

We need to prove now expressions (2.4)-(2.6) of Theorem 3.

The following expression is easy to prove:

$$
\frac{\partial^{j}\left((m-n)^{k+1}\right)_{n=m}}{\partial n^{j}}= \begin{cases}0, & j=1,2, \ldots, k, \\ (-1)^{k+1}(k+1) !, & j=k+1 .\end{cases}
$$

If we use L'Hospital's rule in (A.1) $(n \neq m)$, because $H_{m, m, x, y}^{(k+1) * *}$ must be a finit function it is clear that

$$
\frac{\partial^{j}\left(R H_{m, n, x, y}^{(k+1) * *}\right)_{n=m}}{\partial n^{j}}=0, \quad j=1,2, \ldots, k .
$$

Let us introduce now the following family of functions:

$$
Z_{l}^{j}(m)=\frac{\partial^{j}\left(R H_{m, n, x, y}^{l * *}\right)_{n=m}}{\partial n^{j}}, \quad l=1,2, \ldots, \quad j=l, l+1, l+2, \ldots
$$

It is clear from the former paragraph that

$$
Z_{l}^{j}(m)=0, \quad j=1,2, \ldots, l-1
$$


and

$$
H_{m, m, x, y}^{l * *}=\frac{Z_{l}^{j}(m)}{(-1)^{l} l !}, \quad l=1,2, \ldots
$$

proving expression (2.4) of Theorem 3.

Also for $l=1$, using (A.3),

$$
Z_{1}^{j}(m)=\frac{\partial^{j}\left(R H_{m, n, x, y}^{1 * *}\right)_{n=m}}{\partial n^{j}}=\left(D^{1 *(m)}\right)^{\prime(j)}, \quad j=1,2, \ldots,
$$

expression (2.6) of the statement of Theorem 3 is obtained.

Nevertheless, we still have to prove the recursive formula (2.5).

Now, if we substitute expression (A.2) in (A.9),

$$
\begin{aligned}
Z_{l}^{j}(m)= & \frac{\partial^{j}\left(R H_{m, n, x, y}^{l * *}\right)_{n=m}}{\partial n^{j}}=\frac{\partial^{j}\left(R H_{m, n, x, y}^{(l-1) * *}\left(b^{*}(n)-1\right)\right)_{n=m}}{\partial n^{j}} \\
& -\frac{\partial^{j}\left(H_{m, m, x, y}^{(l-1) * *}\left(b^{*}(m)-1\right)(m-n)^{l-1}\right)_{n=m}}{\partial n^{j}} \\
& +\frac{\partial^{j}\left((m-n)^{l-1}\left(D_{x, y}^{l *}(n)-D_{x, y}^{l *}(m)\right)\right)_{n=m}}{\partial n^{j}}, \quad j=l, l+1, \ldots
\end{aligned}
$$

Using Leibniz's theorem for differentiation of a product in (A.11), for $j=l, l+1, \ldots$,

$$
\begin{aligned}
& \frac{\partial^{j}\left(R H_{m, n, x, y}^{(l-1) * *}\left(b^{*}(n)-1\right)\right)_{n=m}}{\partial n^{j}} \\
& =\sum_{i=0}^{j}\left(\begin{array}{c}
j \\
i
\end{array}\right) \frac{\partial^{i}\left(R H_{m, n, x, y}^{(l-1) * *}\right)_{n=m}}{\partial n^{i}}\left(b^{*}(m)-1\right)^{\prime(j-i)} \\
& =\sum_{i=l-1}^{j}\left(\begin{array}{c}
j \\
i
\end{array}\right) \frac{\partial^{i}\left(R H_{m, n, x, y}^{(l-1) * *}\right)_{n=m}}{\partial n^{i}}\left(b^{*}(m)-1\right)^{\prime(j-i)} \frac{\partial^{j}\left((m-n)^{l-1}\left(D_{x, y}^{l *}(n)-D_{x, y}^{l *}(m)\right)\right)_{n=m}}{\partial n^{j}} \\
& =\left(\begin{array}{c}
j \\
l-1
\end{array}\right) \frac{\partial^{(j-(l-1))}\left(D_{x, y}^{l *}(n)\right)_{n=m}}{\partial n^{(j-(l-1)) i}}(-1)^{l-1}(l-1) !
\end{aligned}
$$

because of (A.8); and also in (A.11):

$$
\frac{\partial^{j}\left(H_{m, m, x, y}^{(l-1) * *}\left(b^{*}(m)-1\right)(m-n)^{l-1}\right)_{n=m}}{\partial n^{j}}=0
$$

Finally, we obtain (2.5) of Theorem 3 


$$
\begin{aligned}
Z_{l}^{j}(m)= & \frac{\partial^{j}\left(R H_{m, n, x, y}^{l * *}\right)_{n=m}}{\partial n^{j}}=\sum_{i=l-1}^{j}\left(\begin{array}{c}
j \\
i
\end{array}\right) \frac{\partial^{i}\left(R H_{m, n, x, y}^{(l-1) * *}\right)_{n=m}}{\partial n^{i}}\left(b^{*}(m)-1\right)^{\prime(l-1-i)} \\
& \quad+\left(\begin{array}{c}
j \\
l-1
\end{array}\right)\left(D_{x, y}^{l *}(m)\right)^{\prime(j-(l-1))}(-1)^{l-1}(l-1) ! \\
= & \sum_{i=l-1}^{j}\left(\begin{array}{c}
j \\
i
\end{array}\right) Z_{l-1}^{j}(m)\left(b^{*}(m)-1\right)^{\prime(l-1-i)}+\left(\frac{(-1)^{l-1} j !}{(j-(l-1)) !}\right)\left(D_{x, y}^{l *}(m)\right)^{\prime(j-(l-1))}, j=l, l+1, \ldots
\end{aligned}
$$

\section{References}

Asmussen, S., 1984. Approximations for the probability of ruin within finit time. Scandinavian Actuarial Journal, 31-57.

Blanc, J.P.C., 1991. The power-series algorithm applied to cyclic polling systems. Stochastic models 7, 527-545.

Bohman, H., 1971. Ruin probabilities. Skandinavisk Aktuarietidskrift, 159-163.

Bohman, H., 1974. Fourier inversion-distribution functions-long tails. Scandinavian Actuarial Journal, 43-45.

Bohman, H., 1975. Numerical inversion of characteristic functions. Scandinavian Actuarial Journal, 121-124.

Char, B. et al., 1991. Maple V Library Reference Manual. Springer, New York.

Cramèr, H., 1955. Collective Risk Theory. Jubille Volume of F. Skandia.

Davies, B., Martin, B., 1979. Numerical inversion of the Laplace transform: a survey and comparison of methods. Journal of Computational Physics 33.

Dickson, C., 1989. Recursive calculation of the probability and severity of ruin. Insurance: Mathematics and Economics 8.

Dickson, C., Waters, H., 1992. The probability and severity of ruin in finit and infinit time. ASTIN Bulletin, 22, 2.

Dickson, C., 1993. On the distribution of the claim causing ruin. Insurance: Mathematics and Economics 12.

Dufresne, F., Gerber, H., 1988. The surpluses immediately before and at ruin, and the amount of the claim causing ruin. Insurance: Mathematics and Economics 7.

Frey, A., Schmidt, V., 1996. Taylor Series expansion for multivariate characteristics of classical risk processes. Insurance: Mathematics and Economics 18.

Gaver, D.P., 1966. Operational Research 14, 444-459.

Gerber, H., Goovaerts, M., Kaas, R., 1987. On the probability and severity of ruin. ASTIN Bulletin, 172.

Gradshteyn, I.S., Ryzhik, I.M., 1994. Table of Integrals, Series and Products, 5th ed. Academic Press, New York.

Grandell, J., 1977. A class of approximations of ruin probabilities. Scandinavian Actuarial Journal, 37-52.

Hooghiemstra, G., Keane, M., van de Ree, S., 1988. Power series for the stationary distributions of coupled processor models. SIAM Journal of Applied mathematics 48, 1159-1166.

Iglehart, D.L., 1969. Diffusion approximation in collective risk theory. Journal of Applied Probability 6, 285-292.

Kroese, D.P., Schmidt, V., 1995. Light-traffi analysis for queues with spatially distributed arrivals. Mathematics of Operations Research. 21(1) $135-157$.

Panjer, H.H., 1981. Recursive calculation of a family of compound distributions. ASTIN Bulletin 12, 22-26.

Piessens, R., 1969. New quadrature formulas for the numerical inversion of Laplace transforms. BIT 9, 351-361.

Rieman, M., Simon, B., 1988. Light traffi limits of sojourn time distributions in Markovian queuing networks. Stochastic models 4, $191-233$. Schmidli, H.P., 1992. A general insurance risk model. Doctoral Thesis, ETH, Zürich.

Seal, H., 1971. Numerical calculation of the Bohman-Esscher family of convolution-mixed negative binomial distribution functions. Mitt. Verein. schweiz. Versich.-Mathr. 71, 71-94.

Seal, H., 1974. The numerical calculation of $U(w, t)$, the probability of non-ruin in an interval $(0, \mathrm{t})$. Scandinavian Actuarial Journal, $121-139$.

Seal, H., 1977. Numerical inversion of characteristic functions. Scandinavian Actuarial Journal, 48-53.

Simon, B., 1993. Calculating light traffi limits for sojourn time distributions in open Markovian queuing systems. Stochastic models 9, $213-231$.

Stehfest, H., 1970. Numerical inversion of Laplace transform. Communications of the ACM, 19 (1).

Thorin, O., 1970. Some remarks on the ruin problem in case the epochs of claims form a renewal process. Skandinavisk Aktuarietidskrift, 29-50.

Thorin, O., 1971. Further remarks on the ruin problem in case the epochs of claims form a renewal process. Skandinavisk Aktuarietidskrift $14-38,121-142$

Thorin, O., 1973. The ruin problem in case the tail of a distribution is completely monotone. Skandinavisk Aktuarietidskrift, 100-119.

Thorin, O., 1977. Ruin probabilities prepared for numerical calculation. Scandinavian Actuarial Journal.

Thorin, O., Wikstad, N., 1973. Numerical evaluation of ruin probabilities for a finit period. ASTIN Bulletin VII (2), 138-153.

Usábel, M. A., 1999. A note on the Taylor-series expansion for multivariate characteristics of classical risk processes. Insurance: Mathematics and Economics, 25, 133-142.

Wikstad, N., 1971. Exemplification of ruin probabilities. ASTIN Bulletin VI, Part 2.

Wikstad, N., 1977. How to calculate ruin probabilities according to the classical risk theory. Scandinavian Actuarial Journal. 